\title{
L'eau, l'écrit et la société. Étude statistique sur les champs sémantiques dans les bases de données [CBMA]
}

Mémoire de Master 2 de l'université de Bourgogne, sous la direction d'Eliana Magnani, Dijon, 2010.

\section{Nicolas Perreaux}

\section{(2) OpenEdition}

\section{Journals}

Édition électronique

URL : https://journals.openedition.org/cem/12062

DOI : $10.4000 /$ cem. 12062

ISSN : 1954-3093

\section{Éditeur}

Centre d'études médiévales Saint-Germain d'Auxerre

Édition imprimée

Pagination : 439-449

ISSN : $1623-5770$

\section{Référence électronique}

Nicolas Perreaux, «L'eau, l'écrit et la société. Étude statistique sur les champs sémantiques dans les bases de données [CBMA] », Bulletin du centre d'études médiévales d'Auxerre | BUCEMA [En ligne], 15 | 2011, mis en ligne le 25 août 2011, consulté le 22 septembre 2022. URL : http:// journals.openedition.org/cem/12062 ; DOI : https://doi.org/10.4000/cem.12062

Ce document a été généré automatiquement le 22 septembre 2022.

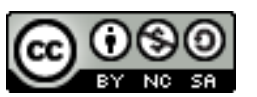

Creative Commons - Attribution - Pas d'Utilisation Commerciale - Partage dans les Mêmes Conditions 4.0 International - CC BY-NC-SA 4.0

https://creativecommons.org/licenses/by-nc-sa/4.0/ 


\section{L'eau, l'écrit et la société. Étude statistique sur les champs sémantiques dans les bases de données [CBMA]}

Mémoire de Master 2 de l'université de Bourgogne, sous la direction d'Eliana Magnani, Dijon, 2010.

\section{Nicolas Perreaux}

Intitulé «L'eau, l'écrit et la société. Étude statistique sur les champs sémantiques dans les bases de données [CBMA] ${ }^{1}$, notre mémoire de master partait d'un constat relativement simple: les chartes, ces documents conservés plutôt en grande abondance, contiennent un nombre extrêmement important de termes relatifs à la description et donc, par extension, à la perception du monde par les médiévaux. Or, il est clair que ces formules, plus ou moins stéréotypées, n'ont presque jamais été considérées comme des informatrices ${ }^{2}$ potentielles en ce qui concerne ce lien entre l'homme médiéval et le mundus, i.e. la Création qui l'entoure. Néanmoins, dès l'orée de l'étude, il est rapidement apparu qu'une telle recherche ne pouvait faire abstraction de plusieurs problématiques plus «classiques» relatives à ce type documentaire: questions de distribution, de géographie et de chronologie. Nous devions donc partir d'un large corpus - la chose étant grandement facilitée par la présence d'un outil exceptionnel au sein du laboratoire ARTeHIS (UMR 5594, CNRS - Université de Bourgogne - Ministère de la Culture) : les CBMA sous Philologic ${ }^{3}$-, étudier initialement sa constitution et son degré d'homogénéité, avant de finalement rejoindre ces considérations d'ordre anthropologique. Fondamentalement, notre recherche s'est donc articulée autour de trois axes :

2 1. La question du développement inégal au sein du système féodal, tout d'abord à travers le problème de la production documentaire différenciée dans l'espace et dans le temps, mais aussi à partir d'une réflexion sur les zones de circulation des formulaires d'actes, zonations ${ }^{4}$ que nous avons proposé d'appeler "aires de scripturalités». 
Partant systématiquement d'observations concernant le champ sémantique de l'eau, cette première partie, intitulée " L'écriture de l'eau » visait avant tout à déterminer une nouvelle chronologie de l'écriture diplomatique, tout en faisant apparaître des différences régionales importantes, que nous avons aussi essayé de catégoriser et d'expliciter en les rapprochant des structures sociales. Le problème fut envisagé à deux échelles bien distinctes, tout d'abord celle de la Bourgogne, mais aussi, avec des sondages plus ponctuels, au niveau d'une partie de l'Europe occidentale - royaume des Francs, Catalogne, Lombardie, Angleterre et Empire.

3 2. Une seconde question largement évoquée fut bien entendu celle du champ sémantique de l'élément aquatique à proprement parler ${ }^{5}$. Quand est-il employé ? Quelle est sa richesse? Aqua, fluvius, rivus, fluviolus, gutta sont-ils utilisés de manière indistincte, comme on le suggère parfois? L'ensemble de ces développements s'inspirèrent abondamment des travaux de Michel Zimmermann sur la Catalogne, en particulier ceux portant sur la question de la dénomination et de la localisation (chapitres "Situer et décrire / dire et inscrire : la mémoire et les cours d'eau » et "L'attention des hommes à l'élément aquatique ») ${ }^{6}$. Cette question a ainsi permis de nous interroger sur les catégories et la typologie attachées au champ sémantique de l'élément aquatique, en particulier avec une mise en parallèle des chartes, d'une part, et des textes d'Isidore de Séville puis de Raban Maur (De Universo) d'autre part: "Aquarum naturae multa est diversitas ${ }^{7}$ ", écrit justement le premier de ces auteurs.

4 3. Enfin, un axe d'ordre plus anthropologique, inspiré, entre autres et pour ce champ de recherche, par les travaux de Claude Lévi-Strauss ${ }^{8}$, de Maurice Godelier ${ }^{9}$ et de Philippe Descola ${ }^{10}$, où l'on s'est interrogé sur l'articulation du champ sémantique de l'eau, non seulement en ce qui concerne sa structuration interne, mais aussi à propos des liens qu'il entretenait avec les autres groupes du lexique. Cette dernière partie, intitulée « Le monde de l'élément aquatique ", a permis de déterminer les principales valences que pouvait prendre l'élément aquatique dans le système de représentation de l'Occident médiéval (lien entre aqua et scriptura par exemple) et de comprendre en quoi elles pouvaient nous éclairer sur le rapport que ces collectifs humains - par ailleurs fortement différenciés - entretenaient avec le monde qui les entourait. Elle fut aussi l'occasion de s'interroger sur le rôle de l'analogie au sein de l'Occident médiéval, non seulement en ce qui concerne les pratiques de l'écrit (omniprésence de formules stéréotypées construites, déconstruites, copiées), mais aussi à propos de son influence sur la structuration et la création du sens au Moyen Âge carolingien et central.

\section{Production documentaire et développement inégal}

5 Dans un premier temps, l'emploi de la statistique a donc permis de mettre en lumière des rythmes de production documentaire intensément scindés, non seulement au sein de la Bourgogne, mais aussi à l'échelle de l'Europe. Ici, nous avons eu recours à différentes bases de données, de tailles variables : les CBMA bien entendu, celle des originaux de l'Artem ${ }^{11}$, mais aussi d'autres fonds numérisés, disponibles grâce à la générosité d'un grand nombre de chercheurs et professeurs, faisant ainsi intervenir volontairement un jeu d'échelles complexe.

6 L'analyse, recourant abondamment à des techniques de fouille de données - Data Mining et Text Mining -, en particulier l'analyse factorielle des correspondances et l'analyse en composantes principales, a permis de montrer que la Bourgogne méridionale possédait 
un «excédent documentaire " très important pour le $\mathrm{x}^{\mathrm{e}}$ et la première moitié du $\mathrm{XI}^{\mathrm{e}}$ siècle - au-delà même du cas clunisien -, la partie septentrionale de la région voyant quant à elle plutôt arriver des chartes aux XII $^{\mathrm{e}}$ et $\mathrm{xIII}^{\mathrm{e}}$ siècles (moment où elles deviennent par ailleurs relativement rares dans le sud de cet espace). Ces constatations furent également l'occasion d'explorer les rapports diplomatiques qu'entretenait cluny avec certains scriptoria proches : Savigny, Sauxillanges, Saint-André-le-Bas de Vienne, mais surtout le chapitre Saint-Vincent de Mâcon, dont on a cherché à montrer le rôle clé dans la mise en place de certaines formules omniprésentes dans le Cluny des premiers temps; en particulier à travers l'emploi d'un formulaire de description de parcelles endémique de cette zone : «a mane [...] a medio die [...] a sero [...] ». L'ensemble de ces observations nous a alors permis de revenir sur la question du lien entre Cluny et Tours et, pour notre part, de défendre l'idée d'une influence diplomatique avant tout locale, au niveau de la zone méridionale de cet espace.

7 Poussant plus loin nos investigations, on a pu montrer que cette double dynamique, qui scindait la Bourgogne et les CBMA en deux ensembles nettement distincts - l'effet de gradient nord/nord-est jouant à quelques dizaines de kilomètres seulement -, s'étendait en fait à l'ensemble du royaume des Francs, avec un grand nombre d'actes anciens dans les zones centrales, mais aussi méridionales : Auvergne, Limousin, Poitou, PACA, Midi-Pyrénées, Catalogne, etc. À l'inverse, les zones du nord de l'espace considéré - Picardie, Champagne, Normandie, etc. -, si elles possèdent un certain nombre d'actes mérovingiens et carolingiens (grosso modo antérieurs à 850), voient avant tout leurs fonds documentaires se remplir pour une période couvrant le $\mathrm{XII}^{\mathrm{e}}$ et le $\mathrm{XIII}^{\mathrm{e}}$ siècle. En définitive, on propose de rapprocher la production documentaire de plusieurs zones: Bourgogne, Auvergne et Rhône-Alpes, qui forment ainsi un premier groupe très proche, se développant très rapidement aux $\mathrm{x}^{\mathrm{e}}$ et $\mathrm{XI}^{\mathrm{e}}$ siècles, suivis de peu par le Poitou, le Limousin, la Provence mais aussi la Catalogne. L'avance de l'espace méridional se réduit quelque peu dans les zones du sud et sud-ouest, qui accusent parfois un certain retard, de plus en plus intense à mesure que l'on va vers l'ouest : Languedoc, Midi-Pyrénées et surtout Aquitaine. Le nord semble lui aussi connaître plusieurs rythmes, avec des espaces à développement plus rapide (Pays-de-la-Loire, Centre par exemple) et d'autres clairement plus en retard (Champagne, Picardie, etc.). Un phénomène de production documentaire inégale donc, que nous avons jugé bon, en première approximation, de rapprocher d'autres éléments caractéristiques de la société médiévale, en particulier architecturaux (roman/gothique). D'une manière générale, les chartes furent envisagées comme des traces de la dynamique sociale, traces qui montrent qu'au-delà des pertes, il existe une véritable cohérence, une logique globale, dans la structure des fonds actuellement conservés ${ }^{12}$.

Cette première analyse des zonations a ensuite été confirmée par l'étude de la circulation de formes/lemmes et autres formules spécifiques du champ sémantique de l'eau. De fait, nombre de termes issus de ce Wortfeld sont spécifiques à certaines régions, plus ou moins vastes, et permettent de (re)construire une "carte théorique " et chronologique de la diffusion de ces mots; une carte qui ne doit sans doute rien à la fantaisie ou au prétendu arbitraire des scribes. C'est le cas des lemmes molinum, fluviolus ou imber, mais aussi celui de la formule "Sicut aqua extinguit ignem, ita elemosinam extinguit peccatum " - omniprésente pour la Bourgogne à Cluny, SaintVincent de Mâcon, Paray-le-Monial, mais strictement absente des corpus du nord de cet espace. Nous avons par la suite étudié sa répartition dans près de 80000 chartes 
indexées, confirmant les remarques ci-dessus à propos des zonations et de l'opposition nord/sud. Ces questionnements ont été menés sur la totalité des chartes à notre disposition, en tenant compte de la difficulté qu'il y a à prendre en considération à la fois la dimension spatiale mais aussi chronologique de ce type de problèmes (il s'agit de biais générés par l'hétérogénéité des corpus, mais une hétérogénéité structurelle - et dont on cherchait précisément à montrer la logique structurale -, et pas seulement fonctionnelle ou méthodologique). Différents outils statistiques ont alors mis en œuvre, afin de s'assurer que les phénomènes observés n'étaient pas générés par de « simples " effets de corpus : regroupement de chartes en paquets égaux ${ }^{13}$, mais aussi Data Mining et analyses factorielles. Il ressort de cette double analyse - production documentaire, diffusion des syntagmes spécifiques - qu'il existait dans l'ensemble de l'espace étudié différentes zonations, très nettes, s'emboîtant les unes avec les autres, dans un jeu d'échelles complexe. Nous voyons ainsi la Bourgogne du sud se raccrocher nettement à la région rhodanienne, à l'Auvergne et au Poitou... mais aussi, d'une manière plus large, à la Provence et à la Catalogne. À l'inverse, la Bourgogne du nord, à travers des éditions telles que le Cartulaire général de l'Yonne ${ }^{14} \mathrm{ou}$ encore les Chartes et documents de SaintBénigne de Dijon ${ }^{15}$, se rapproche nettement des productions d'une large part de l'île-deFrance ${ }^{16}$, du nord-est, de la Normandie, etc. Ce faisceau d'indices permet de conclure non seulement à l'existence de pratiques d'écritures résolument distinctes, aux différences stables sur le temps long (plusieurs siècles), mais aussi à la persistance de structures sociales clairement différenciées et auparavant rarement mises en valeur. Lors de cette réflexion initiale, deux concepts ont abondamment guidé notre réflexion : celui d'échelle et celui de seuil.

\section{L'écriture de l'eau : chronologie, espace, mouvement}

9 La seconde et la troisième parties furent alors consacrées à l'étude du champ sémantique de l'eau proprement dit, dans les chartes en particulier. Ici, les travaux d'Alain Guerreau, mais aussi ceux de Michel Zimmermann servirent - nous l'avons déjà mentionné - de point de départ. Dans un premier temps, il a fallu reconstruire la liste des lemmes composant le champ sémantique de l'eau - opération fastidieuse mais nécessaire -, à partir des sources (chartes et Patrologie Latine), mais aussi à partir des dictionnaires de latin médiéval. Au cours de cette étape, nous avons pu réaliser que le champ sémantique était non seulement passablement présent dans les textes hagiographiques et exégétiques, mais également dans les documents diplomatiques, sous des formes extrêmement variées même chez ces derniers, depuis la présence dans des formules de préambules ("Ecclesia insuper Christi undique adversitatum procellis irruentibus divini auxilii anchora firmata ad ceternee securitatis littus tendens vix periculum evadat naufragii, hanc quam prodixi ecclesiam indignam alieni cognovi amminiculi. » dans une charte du fonds d'Autun ${ }^{17}$ ), jusqu'à la désignation des cours d'eau.

10 Cette seconde partie nous a aussi permis de déterminer puis de nous intéresser aux liens entre ce champ sémantique et d'autres proches, en particulier ceux de l'espace et du mouvement. En effet, une étude plus approfondie des associations entre le mot aqua et les termes désignant l'espace (associations par cooccurrences telles usque aquam, sub aquam, ultra aquam) montre qu'elles sont trois fois plus présentes après 1030 qu'avant cette période. Plusieurs méthodes furent d'ailleurs mises en place afin de quantifier les évolutions de ces vocables. De fait, travailler sur de si larges corpus pose d'innombrables 
problèmes d'ordre statistique mais aussi théorique, la masse n'arrangeant rien ou peu à l'affaire. Dans le cas présent, nous avons eu recours à deux techniques - souvent combinées -: d'une part, l'utilisation assez massive de logarithmes, permettant de limiter fortement divers effets liés à l'hétérogénéité des corpus, d'autre part, le comptage des formes à partir de paquets de chartes égaux. Cette dernière méthode, qui consiste à "découper" la chronologie en plusieurs moments contenant autant de chartes les uns que les autres - à quelques pour cents près -, permet de s'affranchir de la plupart des effets de corpus et de comparer des nombres d'occurrences dans des ensembles de mots relativement égaux.

11 Ainsi donc, on a pu vérifier une explosion du vocabulaire spatial passé 1030 dans le sud de la Bourgogne, un peu plus tard dans le nord. Cette augmentation de l'association entre eau et espace s'accompagne, en parallèle, de la diminution de l'association toujours par cooccurrences - entre eau et mouvement (aqua vergit, aqua discurrente, etc.). Tout se passe donc comme si, entre 1030 et 1080, l'eau était de plus en plus associée à l'espace - avec l'emploi beaucoup plus fréquent des formes à l'accusatif, type aquam, qui permettent de désigner, de montrer - tandis que le mouvement prenait une place de plus en plus faible dans les documents diplomatiques. Cette dynamique est accompagnée par l'emploi de plus en plus concret des termes du champ sémantique. Une analyse du sens donné à aqua, en fonction de son degré d'abstraction (très abstrait : préambules et formules stéréotypées - très peu abstrait/concret : hydronymes et toponymes) montre que pour cette même période - 1030-1080 donc -, on note un très net infléchissement de la valeur attribuée aux termes employés et un passage d'un moment où l'eau est essentiellement utilisée de manière abstraite, inquantifiable, à un moment où on l'emploie avant tout comme une chose concrète et définie. D'un point de vue social, il nous parait intéressant de rapprocher toutes ces observations concordantes $\mathrm{du}$ concept d'encellulement proposé par Robert Fossier ${ }^{18}$. Plus intéressant encore, il semble que cette émergence de l'espace connaît d'amples variations - ce qui nous ramène bien entendu à notre réflexion sur le développement inégal - d'une région à une autre, ne serait-ce qu'entre Bourgogne du sud et Bourgogne du nord. Le mémoire fut ainsi l'occasion de s'interroger sur l'opérabilité des modèles historiographiques du système féodal et de montrer - en substance - que l'opposition, souvent avancée comme canonique, entre la chronologie proposée par Georges Duby et celle avancée par Marc Bloch est illusoire, les deux étant en fait complémentaires, l'une et l'autre fonctionnant en fait à des échelles différentes, mais qui correspondent à des phénomènes de mise en place de structures similaires : en Bourgogne du sud pour le premier; à l'échelle de l'Europe et en moyenne pour le second. À notre sens, les évolutions bien réelles d'effectifs relatifs à des champs sémantiques tels que l'eau, l'espace, le mouvement, sont des indices précieux de la mise en place de nouvelles structures matérielles, mais aussi d'un nouveau rapport au monde - en disant cela, nous pensons bien évidemment aux travaux de Maurice Godelier sur la part « idéelle du réel ${ }^{19}$.

12 Poursuivant sur cette voie, nous avons aussi «testé » l'évolution que connaissaient les vingt lemmes les plus courants du champ sémantique de l'eau, en bloc, en recourant cette fois aux coefficients de corrélations. Le corpus a alors été découpé en quatre parties contenant un nombre égal de chartes, pour ensuite dénombrer les occurrences de chacun des vingt lemmes dans ces quatre moments. Il suffisait ensuite de calculer les coefficients de corrélations sur ces quatre colonnes/périodes pour observer celles qui étaient les plus liées : à nouveau, sans guère de surprise désormais, on note une rupture 
très nette entre les années 1030 et 1080 . Cette flexion très forte est d'ailleurs d'autant plus remarquable qu'elle se produit en seulement quelques décennies et s'oppose, par exemple, à la grande stabilité des effectifs repérée pour les périodes précédentes. L'ensemble de cette réflexion a ainsi permis de revenir sur la question de la «mutation féodale ", le champ sémantique de l'eau étant, en effet, un indicateur relativement neutre - a contrario des lemmes tels que miles ou feodum sur lesquels a porté le débat jusque-là - de l'évolution remarquable, certes dépendante des régions, de l'ensemble du vocabulaire entre le début $d u \mathrm{XI}^{\mathrm{e}}$ siècle et la fin $\mathrm{du} \mathrm{XII}^{\mathrm{e}}$ : une évolution d'autant plus frappante qu'à nouveau son intensité n'a d'égale que sa rapidité, une fois le tournant lexical amorcé.

\section{Le monde de l'élément aquatique : entre espace et non-espace ${ }^{20}$}

13 La dernière partie de notre travail - volontairement inspirée par les travaux de Philippe Descola sur la question des rapports à l'altérité et au monde - fut consacrée à l'étude de la structuration du champ sémantique de l'eau dans la Patrologie Latine. Partant d'une citation de Pierre le Vénérable relevée par Dominique Iogna-Prat "Aspice aquam in glaciem commutatam, glaciem in crystallum alteratam, et cernes in his aperte substantias permutari, formas substantiarum seu species reservari ${ }^{21}-$, on a voulu savoir quelle valence était attribuée à chaque élément du champ et surtout quel était son dénominateur commun (chapitre «Les valences du champ sémantique de l'eau: comparatisme et formalisation»). On a pour cela réalisé une série d'analyses factorielles sur un tableau de cooccurrences généralisées pour vingt-et-un mots-clés relatifs au champ - en fait vingt-et-une espèces de l'élément aquatique : aqua, fons, lacus, rivus, unda, oceanus, fluvius, mare, diluvium, lacrima, abyssus, nubes, pluvia, imber, ros, gutta, nix, etc. Différents traitements ont alors été appliqués à ces vastes tableaux de cooccurrences, en particulier en employant un «codage logique» ou "codage disjonctif complet $»^{22}$.

14 Très nettement, ces expériences attestent que le champ sémantique est marqué par une forte opposition entre ce que nous avons nommé "espèces terrestres » et " espèces célestes", chacune de ces grandes catégories s'organisant de part et d'autre des analyses factorielles. Nous avons ainsi pu montrer de manière automatique et non $a$ priori que c'était bien l'opposition du charnel et du spirituel, si bien relevée par Anita Guerreau-Jalabert, qui gouvernait la structuration du champ sémantique de l'élément aquatique et ceci sur le temps long. Cette première clé sémantique déterminée, elle fut appliquée à plusieurs cas précis, afin de vérifier son opérabilité.

Dès lors, une analyse plus « qualitative » a été engagée, recourant abondamment au $D e$ miraculis de Pierre le Vénérable ${ }^{23}$ (qui contient plusieurs passages renvoyant à la question de l'articulation entre eau et espace), mais aussi à l'introduction du Cartulaire de l'abbaye d'Uzerche ${ }^{24}$, ou encore au préambule clunisien - que l'on retrouve par ailleurs au même moment chez Odon de Cluny - relatif à la parabole de Lazare et du pauvre ${ }^{25}$ - et dont l'articulation des principaux éléments a par la suite été analysée : eau / feu / pauvreté / richesse / intériorité / extériorité / immatériel / matériel / sein d'Abraham / enfer / mie de pain / goutte d'eau/haut (céleste) / bas (infernal). L'ensemble de ces investigations, recourant de manière secondaire à des sources iconographiques ${ }^{26}$, permit de montrer que l'eau était presque systématiquement 
associée : 1. à la pauvreté (et par extension au don). 2. au rejet de l'extériorité et du matériel. 3. à l'intériorité (spirituelle). En parallèle, d'autres investigations ont été poursuivies sur la nature (i.e. les qualités) de l'eau baptismale en tant qu'élément de transitus (chapitre: "La double nature des eaux: correspondances et visio dans le sacrement du baptême »).

D'une manière plus large, les multiples valences - essentiellement positives - attribuées à l'eau (et à son champ sémantique), qui agit donc un peu comme un «joker » au sein du système de représentation, nous ont permis de revenir sur l'opérabilité du modèle des ontologies et du régime analogique tels qu'ils sont définis par Philippe Descola. Cette réflexion fut ainsi l'occasion de revenir sur la préface des Historiae de Raoul Glaber à propos de la Divine quaternité ${ }^{27}$ et de tenter de montrer le rôle fondamental qu'y jouent non seulement l'élément aquatique, mais aussi l'analogisme généralisé. En définitive, l'eau, élément clé du passage au sein du système de l'Occident féodal - aussi bien au plan idéel que social -, tissant sans cesse des liens avec la plupart des autres éléments du système de représentation, est, à notre sens, le paradigme même de ce fonctionnement par hiérarchisation et analogie. L'hypothèse posée est donc que les inégalités d'écriture et de production documentaire à l'échelle européenne traduisent non seulement le développement inégal du système féodal - phénomène qui se note par exemple dans l'emploi de termes, de discours ${ }^{28}$, très nettement différenciés - mais aussi des rapports (sémantiques) au monde et à l'autre relativement dissemblables dans le temps et dans l'espace, le tout cohabitant au sein d'un système pourtant cohérent. C'est cette hypothèse globale que nous souhaitons explorer lors d'une thèse en cours ${ }^{29}$, en recourant abondamment aux techniques de fouilles de données (Data/Text Mining) et en employant cette fois un corpus documentaire déjà réuni par nos soins, comprenant, outre les textes théologiques plus de 150000 chartes numérisées.

\section{NOTES}

1. Nous tenons à remercier chaleureusement Eliana Magnani et Alain Guerreau pour leur relecture attentive et pour leurs conseils lors de la rédaction de ce résumé de notre mémoire. Chantal Palluet, Pierre Bonnerue et Daniel Russo ont participé à la relecture finale de cet article : qu'ils en soient remerciés eux aussi. Pour des raisons évidentes de concision, les références bibliographiques de l'article ont été limitées au strict minimum.

Ce mémoire n'aurait pas vu le jour sans l'aide attentive et le soutien constant de notre directrice Eliana Magnani, que je tiens à remercier avant tout vivement. Nous avons en outre bénéficié de l'aide exceptionnelle d'Alain Guerreau, jamais avare de ses idées et de son temps: ce modeste travail lui doit plus que je ne peux le dire. Nous tenons aussi à remercier Marie-José GasseGrandjean et Alain Rauwel pour leur relecture et leurs conseils assidus tout au long du travail. De la même manière, plusieurs discussions avec Daniel Russo, Anita Guerreau-Jalabert, Bruno Bon, Sébastien Barret, Bruno Lemesle, Jean-Pierre Garcia, Hervé Mouillebouche et Thomas Labbé ont grandement facilité notre travail sur plusieurs points : qu'ils en soient eux aussi sincèrement remerciés. En outre, ce travail n'eut pas été le même sans les conseils de Maria Hillebrandt et de l'équipe des médiévistes de Münster (Martin Kintzinger, Franz Neiske et Torsten Hiltmann), 
grâce à leur merveilleux accueil sur place pour un séjour de recherche. Nous tenons aussi à remercier Paul Bertrand et Sébastien Barret pour nous avoir permis de présenter les premières conclusions de ce jeune travail à l'International Medieval Congress de Leeds en 2010. D'une manière plus large, notre recherche a bénéficié d'une aide financière substantielle de la part du Conseil régional de Bourgogne. Enfin, ce mémoire a profité de la mise à disposition de corpus que plusieurs professeurs et chercheurs ont accepté de nous transmettre, nous tenons à les remercier vivement pour ce geste désintéressé : il s'agit de Benoît-Michel Tock, de Pierre Baudouin, d'Isabelle Rosé, de Florian Mazel, de Laurent Ripart, de Jean-Baptiste Renault et, enfin, de Michel Lauwers.

2. Le terme "informatrice" est employé à dessein. Il s'agit d'insister sur la dimension interactive de la recherche et sur le fait que la « réaction » des documents n'est jamais passive, mais dépend étroitement du questionnaire qu'on leur applique.

3. À propos des CBMA, voir l'ensemble des articles d'Eliana Magnani et de Marie-José GasseGrandjean dans le Bucema [http://cem.revues.org/]: n 9 (2005), p. 179 [index751.html] ; 11 (2007), p. 163-167 [index1064.html] ; 12 (2008), p. 237-244 [index6962.html] ; 13 (2009), p. 245-251 [index11077.html] ; 14 (2010), p. 197-207 [index11433.html] et p. 273 [index11582.html].

4. Il s'agit d'un concept emprunté à la géographie et à l'écologie scientifique, servant habituellement à désigner l'étendue d'un écosystème, d'un climat ou d'un phénomène naturel en général.

5. J. TRIER, Der Deutsche Wortschatz im Sinnbezirk des Verstandes: Die Geschichte eines sprachlichen Feldes, Band 1: Von den Anfängen bis zum Beginn des 13 Jahrhunderts, Heidelberg, 1931; ID., «Über Wort- und Begriffsfelder ", in L. scHмIDT (éd.), Wortfeldforschung: zur Geschichte und Theorie des sprachlichen Feldes, Darmstadt, 1973, p. 1-38 ; A. GUERREAU, « Le champ sémantique de l'espace dans la vita de Saint Maieul (Cluny, début du XI ${ }^{\mathrm{e}}$ siècle) ", Journal des savants, 1997, p. 363-419; ID., L'avenir d'un passé incertain. Quelle histoire du Moyen Âge au XXI" siècle ?, Paris, 2001 ; ID., " "Textus" chez les auteurs latins du XII ${ }^{\mathrm{e}}$ siècle ", in L. KUCHENBUCH et U. KLEINE (éd.), “Textus" im Mittelalter, Göttingen, 2005, p. 149-178; A. GUERREAU-JALABERT, « Parole/Parabole : analyse d'un champ lexical et sémantique ", in R.-M. DESSI et M. LAUWERS (éd.), La parole du prédicateur, Nice, 1997, p. 311-339; A. GUERREAU-JALABERT et B. BON, «Pietas: réflexions sur l'analyse sémantique et le traitement lexicographique d'un vocable médiéval», Médiévales, 42 (2002), p.73-88; EID., «Le trésor au Moyen Âge : étude lexicale ", in L. BURKART, P. CORDEZ, P.-A. MARIAUX et Y. POTIN (dir.), Le trésor au Moyen Âge. Discours, pratiques et objets, Florence, p. 11-32.

6. M. ZIMMERMANN, "Glose, tautologie ou inventaire? L'énumération descriptive dans la documentation catalane $\mathrm{du} \mathrm{x}^{\mathrm{e}}$ au $\mathrm{XII}^{\mathrm{e}}$ siècle ", Cahiers de linguistique et de civilisation hispaniques médiévales, 14-15 (1989-1990), p. 309-338; ID., «Les noms de la route et du chemin dans la Catalogne médiévale ", in C. DESPLAT (dir.), Terres et hommes du sud. Hommage à Pierre Tucoo-Chala, Biarritz, 1992, p. 387-413 ; ID., Écrire et lire en Catalogne (IX ${ }^{e}-X I I^{e}$ siècle), Madrid, 2003.

7. ISIDORUS HISPALENSIS, Etymologiae. Libro XIII, De mundo et partibus, éd. PL 82, col. 73-0728C, ici col. 482A-482B ; édition plus récente dans G. GASPAROTTo (éd. et trad.), Etymologiae, Libro XIII, De mundo et partibus, Paris, 2004.

8. Ici en particulier : C. LÉVI-STRAUSS, «La geste d'Asdiwal », in Anthropologie structurale II, Paris, 1973, p. 175-233 [1re édition de l'article dans École pratique des hautes études. Section des sciences religieuses. Annuaire 1958-1959, Paris, 1958, p. 3-43 ; aussi in Les temps modernes, $\mathrm{n}^{\circ} 179$, mars 1962] ; ID., La Pensée sauvage, Paris, 1962.

9. Cf. infra.

10. P. DESCOLA, Par-delà nature et culture, Paris, 2005.

11. B.-M. TOCK (dir.), M. COURTOIS, P. DEMONTY et M.-J. GASSE-GRANDJEAN (réalisé par), La diplomatique française du haut Moyen Âge: inventaire des chartes originales antérieures à 1121 conservées en France, 2 vol., Turnhout, 2001. 
12. Certaines de nos expériences plus récentes tendent à montrer - après un dépouillement assez large de cartulaires - que la structure des originaux conservés est extrêmement proche de celle de l'ensemble des documents édités. Un point qui devra être exploré et vérifié lors d'une thèse en cours.

13. Cette pratique, clairement inspirée par les travaux et remarques d'Alain Guerreau (en particulier son cours intitulé Statistique pour historiens, professé à l'École des chartes en 2003-2004, disponible en ligne: http://elec.enc.sorbonne.fr/statistiques/stat2004.pdf), part d'une série de constatations sur la complexité des distributions lexicales. À la lecture de quelques ouvrages de référence, on comprend aisément qu'il est strictement impensable de se baser sur les effectifs bruts ou même - c'est sans aucun doute pire - sur des pourcentages, afin d'effectuer des comparaisons entre corpus documentaires ou même entre différentes périodes. La plupart des outils restent donc à inventer et ces deux années de recherches furent aussi l'occasion d'en appliquer et de tester l'efficacité d'un certain nombre. Outre le cours mentionné ci-dessus, quelques jalons essentiels dans : H. R. BAAYEN, Word Frequency Distributions, Dordrecht, 2001 (Text, Speech and Language Technology $\left.n^{\circ} 18\right)$; S. EVERT, The statistics of word cooccurrences: Word pairs and collocations, Stuttgart, 2005 ; A. GUERREAU, « Pourquoi (et comment) l'historien doit-il compter les mots? », Histoire \& mesure, 4 (1989), p. 81-105 ; B. MANDELBROT, Les objets fractals. Forme, hasard et dimension, Paris, 1975 ; ID., Fractales, hasard et finance, Paris, 1997 (en particulier le chapitre 4, "Aléas du discours »).

14. M. QuANTin (éd.), Cartulaire général de l'Yonne, 2 vol. et supplément, Auxerre, 1854-1860 et 1873.

15. G. CheVRIER, M. ChAUME, R. Folz et J. MARILIER (éd.), Chartes et documents de Saint-Bénigne de Dijon : prieurés et dépendances, des origines à 1300, 2 vol., Dijon, 1943-1986.

16. Nous avons utilisé la base de données créée par l'École des chartes, qui contient plusieurs milliers d'actes franciliens.

17. A. DE CHARMASSE (éd.), Cartulaire de l'Église d'Autun, Paris, 1865-1900, nº $14-1033$.

18. R. FOSSIER, Enfance de l'Europe. Aspects économiques et sociaux, 2 vol., Paris, 1982.

19. M. GODELIER, « La part idéelle du réel », L’Homme, 18 (1978), p. 155-187 ; ID., L'idéel et le matériel Pensée, économies, sociétés, Paris, 1984.

20. A. GUERREAU, « Le champ sémantique... », op. cit.

21. Petrus Cluniacensis, Adversus Petrobrusianos haereticos, éd. PL 189, col. 719-0850D, ici col. 805A-805B ; voir aussi l'édition plus récente de J. FEARNs (éd.), Contra Petrobrusianos hereticos, Turnhout, 1968 (CCCM, 10) ; passage mentionné dans D. IOGNA-PRAT, Ordonner et exclure. Cluny et la société chrétienne face à l'hérésie, au judaïsme et à l'islam (1000-1150), Paris, 1998, p. 148.

22. L'ensemble du tableau a été converti en binaire selon une règle du type: présence de la cooccurrence pour le mot-clé $=1$; absence $=0$.

23. D. BOUTHILliER (éd.), De Miraculis libri duo, Turnhout, 1988 (CCCM, 83) ; D. BOUTHILLIER et J.P. TORRELl (trad.), Les Merveilles de Dieu, Paris/Freiburg, 1992.

24. J.-B. ChampeVAl (éd.), Cartulaire de l'abbaye d'Uzerche (Corrèze) du X $X^{e}$ au XIVe siècle, Paris, 1901.

25. "[...] pro micis panis quas ulceroso Lazaro negavit, stillam aquae in inferno ardens desideravit [...]» dans A. BERNARD et A. BRUel (éd.), Recueil des chartes de l'abbaye de Cluny, 6 vol., Paris, 1876-1903, t. $1, n^{\circ} 269$, en 926 . Nous avons aussi consacré plusieurs pages à cette charte, rédigée par un clerc présent à Saint-Vincent de Mâcon et sans doute proche de l'évêque de cette même ville, qui eut un impact remarquable sur les pratiques de l'écrit clunisien. Elle montre que les clunisiens ont avant tout puisé dans un répertoire de formules locales et ceci dès leurs origines (mais comment aurait-il pu en être autrement?).

26. J. BASCHET, Le sein du père. Abraham et la paternité dans l'Occident médiéval, Paris, 2000. 
27. RODULFUS GLABER, Historiarum libri quinque, éd. et trad. J. FRANCE, Rodulfi Glabri. Historiarum libri quinque. Vita domni Willelmi abbatis (The life of St William, by the same author), Oxford, 1989 ; aussi éd. PL 142, col. 611-698 ; traduction française in M. ARNOUX (éd.), Histoires, Turnhout, 1996.

28. M. FOUCAULT, Les mots et les choses, Paris, 1966 ; ID., L'archéologie du savoir, Paris, 1969.

29. Sous la direction d'Eliana Magnani et de Daniel Russo, ainsi qu'avec l'aide d'Alain Guerreau.

INDEX

Mots-clés : base de données, champ sémantique, eau, écrit, étude statistique, société 